\title{
Educação permanente em saúde e as Diretrizes Curriculares Nacionais para Educação das relações étnico-raciais e para ensino de História e Cultura Afro-Brasileira e Africana Permanent health education and National Curriculum Guidelines to the education of ethnic-racial relations and teaching of Afro-Brazilian and African History and Culture
}

\section{Rosana Batista Monteiro}

Universidade Federal de São Carlos. Departamento de Ciências Humanas e Educação. Sorocaba, SP, Brasil.

E-mail: rosanabmonteiro®ufscar.br

\section{Correspondência}

Universidade Federal de São Carlos - Rodovia João Leme dos Santos (SP-264), km ıo,

Bairro do Itinga. Sorocaba, SP, Brasil. CEP 18052-780.

\section{Resumo}

Este texto é um ensaio sobre algumas contribuições que o curso de especialização em Saúde da Mulher Negra pode ter produzido para a abordagem dos conteúdos relacionados nas Diretrizes Curriculares Nacionais para a Educação das Relações Étnico-raciais e para o Ensino de História e Cultura Afro-brasileira e Africana, bem como em atenção à Política Nacional de Saúde Integral da População Negra, com destaque para a educação permanente. A partir de breve contextualização sobre o debate no campo das relações raciais e ações afirmativas no Brasil passamos a apresentar a organização e funcionamento do curso, destacando seus objetivos, conteúdos, estratégias. Com foco nos profissionais da saúde, o curso contou com significativa presença de profissionais da educação básica pública, municipal e estadual, das cidades de São Luís e Pinheiro, no Maranhão, implicando a discussão do tema saúde da população negra (especialmente da mulher negra) no âmbito da escola. 0 curso propiciou a elaboração de projetos de pesquisa relacionados diretamente com o atendimento à saúde de mulheres negras e sua relação com processos formativos de profissionais da área da saúde, bem como educação escolar e saúde de mulheres negras. Pode-se concluir, ainda que provisoriamente, que o curso, na perspectiva da educação permanente, resultou em estratégia eficaz para o desenvolvimento de conteúdos relacionados às DCNERER e, especialmente, de viabilização de im- 
plementação da Política Nacional de Saúde Integral da População Negra, realizadas ou desencadeadas nos serviços de saúde pelos cursistas.

Palavras-chave: Educação Permanente; Relações Raciais; População Negra; Saúde da Mulher.

\section{Abstract}

This text is an essay about the contributions that the Black Woman's Health specialization course as a strategy of Permanent Education can have produced to the approach of the contents related in the National Curriculum Guidelines to the Education of ethnic-racial relations and teaching of Afro-Brazilian and African History and Culture as also attending to the National Policy for Integrated Health of the Black Population. From a brief background on the field of racial relations and affirmative actions in Brazil, we present the course organization and functioning, highlighting its objectives, contents, strategies. Focusing on health professionals, the course was attended by professionals from basic education, from the cities São Luis and Pinheiro, in the state of Maranhão, Brazil, implying the discussion of the theme black population (especially black women) health, in the context of health and school services. Although few participants have completed their papers, we observed that the course led to the development of research projects directly related to the black women health care and to health professionals' education and formative processes. It can be concluded, even temporarily, that the papers reveal that the course, from the perspective of lifelong learning, resulted in an effective strategy for studying the content related to DCNERER and the conditions of the attendance offered to black women in health services, and also the propositions of strategies and actions involved in the implementing process of National Policy for Integrated Health of the Black Population.

Keywords: Permanent Education; Racial Relations; Black Population; Women's Health.

\section{Introdução}

Os primeiros anos do século XXI inauguram uma nova e profícua fase em relação à definição de políticas de ação afirmativa voltadas à população negra no Brasil. No entanto, ainda não é possível afirmar que, na prática, as políticas tenham se efetivado. Encontram-se ainda em processo de implementação, algumas em estágio mais avançado, dependendo da área - educação, saúde, direito, segurança, dentre outras. Há políticas e programas com maior ou menor investimento de recursos públicos, mas todos, de alguma maneira, encontram resistências relacionadas principalmente ao racismo institucional impregnado nos equipamentos públicos. Em 2013, pouco tivemos a comemorar nos dez anos da aprovação da conhecida Lei 10.639/2003, que determinou a alteração do texto da Lei 9.394/1996, Lei de Diretrizes e Bases da Educação Nacional (LDB), com inclusão dos artigos 26-A e 79-B. Os referidos artigos se referem, respectivamente, à obrigatoriedade do ensino de História e Cultura afro-brasileira e africana nos currículos das escolas de ensino fundamental e médio e à inclusão do dia 20 de novembro, Dia da Consciência Negra, no calendário escolar. Muitas escolas brasileiras ainda não incluíram tais conteúdos em seus currículos, não enfrentam a necessária reeducação das relações étnico-raciais, de forma a romper com práticas discriminatórias vivenciadas cotidianamente por estudantes e profissionais da educação.

Em 2004 foram aprovados o Parecer CNE 03/2004 e a Resolução CNE/CP o1/2004, que instituíram as Diretrizes Curriculares Nacionais para a Educação das Relações Étnico-raciais e para o Ensino de História e Cultura Afro-Brasileira e Africana (DCNERER), regulamentando os artigos citados anteriormente.

As DCNERER afirmam que, prioritariamente, os cursos de formação de professores estão obrigados a incluir os conteúdos relacionados à história e à cultura afro-brasileira e africana, o que implica a atenção de alguns cursos da área da saúde, com destaque para o de enfermagem, que se trata de uma licenciatura. Infelizmente, os cursos da área 
da saúde pouco ou nada têm feito no sentido de considerar o tema em questão como conteúdo pertinente à formação dos novos profissionais da saúde - curiosamente em um país com metade da população autodeclarada negra.

Ainda nesse período, em decorrência da mobilização de ativistas e/ou profissionais ligados à saúde da população negra, elabora-se documento relativo à saúde da população negra que, em 2009, depois de longo processo de pactuação, foi finalmente aprovado na forma de Política Nacional de Saúde Integral da População Negra (PNSIPN), Portaria GM/MS nº 992/2009. Não cabe neste texto discorrer sobre esse processo e documento, mas vale lembrar que está em estreita articulação com a Política Nacional de Saúde delimitada no Sistema Único de Saúde (SUS). A implantação do SUS em atenção aos seus princípios - universalidade, integralidade, equidade, descentralização, participação social - somente poderão ser efetivados se, dentre outras ações, atenderem às especificidades da saúde da população negra.

Em documento, por nós elaborado, sobre a PNSIPN e Educação Permanente em Saúde apresentamos "propostas de conteúdos, metodologias e tecnologias voltadas à inserção da Política Nacional de Saúde Integral da População Negra nos processos de educação permanente em saúde considerando quatro dimensões: a gestão, a atenção à saúde, a formação de profissionais e o controle social"'. A educação permanente, no campo da saúde pública, é compreendida enquanto um conceito pedagógico que articula as dimensões mencionadas que, ampliadas pela reforma Sanitária Brasileira, passam a articular as relações entre docência e atenção à saúde, formação e gestão, desenvolvimento institucional e controle social em saúde.

Entendemos que, para dar efetividade ao disposto no SUS e na PNSIPN, esta destaca a importância da Educação Permanente em Saúde. A formação dos profissionais da saúde é estratégia fundamental para se garantir a atenção à saúde da população negra, em especial, das mulheres negras como parte mais vulnerável dessa população. Para que a Educação Permanente atenda aos desafios a que é chamada pela PNSIPN ela deve considerar o exposto nos documentos relacionados às DCNERER, sem o que não se pode atuar de maneira integral frente à complexidade das relações raciais presentes na sociedade brasileira.

Ambas as políticas, a de saúde e a de educação, são instrumentos de combate ao racismo que permeia o tecido social na sociedade brasileira e adentra os equipamentos públicos. Há uma extensa literatura relacionada a esses campos, em separado e em articulação. Muitas pesquisas foram desenvolvidas nos anos recentes, sem contar toda a história de denúncia feita pelo movimento social negro, desde ao menos a década de 1970 (Batista, 2002; 2005; Lopes, 2003; Lopes; Buchalla; Ayres, 2007; Oliveira, 2003; Monteiro, 2010; Batista; Werneck; Lopes, 2012).

Os desafios da saúde pública e da formação acadêmica acessível a todos e de qualidade passa pelo enfrentamento das razões que determinam a produção e reprodução das desigualdades sociais na sociedade brasileira. Dentre esses determinantes sociais, faz-se necessário reconhecer que a resolução das questões de ordem econômica (classes sociais) é insuficiente. As questões de classe precisam se articular às questões de gênero, geração, orientação sexual e raça-etnia. Interessam-nos, neste texto, e no curso aqui analisado, especialmente as implicações do racismo como determinante de desigualdades sociais que, no caso da saúde pública, determinam as condições de nascimento, vida e morte de crianças, jovens, adultos, homens e, especialmente, mulheres negras. Mas se há uma vítima em potencial que é atingida pelas diferentes formas de manifestação do racismo no âmbito da saúde pública, pode-se afirmar também que toda a sociedade, negros e não negros, é afetada direta ou indiretamente por esse fenômeno.

Para que se possa combater o racismo e seus efeitos para a população negra e nãonegra, em primeiro lugar, é necessário reconhecermos sua existência.

1 MONTEIRO, R. B. Orientações e experiências para a implementação da Política Nacional de Saúde Integral da População Negra no âmbito da formação e Educação Permanente em Saúde. Documento elaborado para o Comitê Técnico de Saúde da População Negra/Secretaria de Gestão Estratégica e Participação do Ministério da Saúde. 2011. p. 3. 
Como afirma Kabengele Munanga (2010, p. 170):

o maior problema da maioria entre nós parece estar em nosso presente, em nosso cotidiano de brasileiras e brasileiros, pois temos ainda bastante dificuldade para entender e decodificar as manifestações do nosso racismo à brasileira, por causa das peculiaridades que o diferenciam das outras formas de manifestações de racismo [...] [nazismo, apartheid, segregação racial norte-americana] ecoa dentro de muitos brasileiros, uma voz muito forte que grita; 'não somos racistas, os racistas são os outros'.

O objetivo último deste texto, bem como do curso aqui em análise, à luz das DCNERER e da PNSIPN, é o de combater o racismo que se manifesta, em particular na atenção à saúde da população negra.

\section{o curso: sua proposta, estratégias, objetivos}

O Curso de Especialização em Saúde da Mulher Negra, oferecido pelo Programa de Pós-Graduação em Saúde e Ambisente da Universidade Federal do Maranhão, teve como seus principais articuladores os professores pesquisadores Istvan Vargas (Universidade Federal do Maranhão - UFMA) e Luís Eduardo Batista (Instituto de Saúde/Secretaria do Estado de São Paulo - IS/SES-SP) e Maria de Fátima Oliveira Ferreira (Universidade Federal de Minas Gerais-UFMG) dentre outros. O curso foi financiado com recursos provenientes do Ministério da Saúde e foi oferecido no estado do Maranhão em duas localidades, na capital - São Luís - e no município de Pinheiro.

A proposição do curso e sua realização no estado do Maranhão se devem à constatação de que, de acordo com Cunha (s. d.) "a população negra está exposta a um ciclo de desvantagens cumulativas na mobilidade social intergeracional, fato este que a coloca em posição de maior vulnerabilidade frente a uma série de agravos para sua saúde”. E ainda, de que, sendo o Maranhão um dos estados brasileiros com maior concentração de população negra, apresenta-se como lugar privilegiado para o desenvolvimento necessário de ações de saúde que estejam atentas às especificidades dessa população. A escolha dos municípios onde o curso foi desenvolvido também não foi aleatória, relaciona-se à existência de espaço físico da UFMA adequado a seu desenvolvimento, bem como às necessidades de promoção de formação permanente em saúde em localidades nas quais as estatísticas relacionadas à atenção à saúde da mulher negra demonstram a precariedade do acesso e de atendimento de qualidade, alto índice de morbi-mortalidade, dentre outros.

O projeto do curso em questão destaca que, apesar de a saúde da população negra se apresentar como um "campo de estudos, pesquisas e assistência reconhecido do ponto de vista da legitimidade política, cujas bases científicas são inegáveis”, ele ainda encontra dificuldades para ser incorporado nas práticas de atendimento, no planejamento e execução das políticas públicas de saúde, e mesmo nos espaços de formação dos profissionais de saúde. O curso, nesse sentido, propôs como objetivo geral "formar especialistas em saúde da mulher negra e estimular a iniciação na investigação acadêmica em saúde da mulher negra.” Dentre os objetivos específicos do curso podemos citar:

Fornecer elementos sobre racismo e recorte racial/ étnico na saúde com vistas a estabelecer compreensão e visão críticas anti-racistas quanto aos fatores desencadeadores e determinantes do racismo na atenção à saúde; compreender o impacto do racismo na saúde; estimular práticas inovadoras de atenção com perspectiva de gênero e anti-racista na Rede Pública de Saúde; contribuir para a incorporação pelos centros acadêmicos brasileiros da temática da saúde da população negra; ampliar a consciência sobre as principais questões relativas à saúde da população negra, com ênfase na saúde da mulher negra e na reflexão de como a vivência do racismo e da violência (gênero e racial) repercute na saúde².

2 Projeto Político Pedagógico do Curso de Especialização em Saúde da Mulher Negra. UFMA/Programa de Pós-graduação em Saúde e MEIO ambiente. Maranhão. 2007 
Como se pode observar, os objetivos não apontam para a formação de professores, nem mesmo para o estudo de temas em saúde da população negra/mulher negra relacionados com a educação básica. No entanto, muitos professores se interessaram em participar do curso, tendo em vista que nas salas de aulas convivem com crianças e jovens negras que poderiam se beneficiar dos conhecimentos abordados. Em certa medida, é possível afirmar que os professores também se interessaram pelo curso por ele oferecer uma certificação de especialista. Independentemente da razão que os levaram a ingressar e participar do curso, veremos que este trouxe uma contribuição positiva a profissionais que, inicialmente, não eram entendidos como público-alvo prioritário. Cabe destacar, no entanto, de acordo com o projeto do curso e, consequentemente, a definição do processo de seleção que: "No que se refere ao corpo discente, as inscrições no Curso estão facultadas, do mesmo modo, a profissionais de nível superior de qualquer área de conhecimento (desde que atendidas as condições dispostas no item ‘6. Público-alvo')”3.

O curso foi desenvolvido na forma de módulos concentrados em duas semanas por mês, com encontros presenciais e acompanhamento parcialmente à distância por professores e orientadores. Essa estratégia se deveu especialmente pelo fato de a equipe docente ser de diferentes estados do Brasil, o que determinou a criação de estratégias de planejamento dos deslocamentos constantes dos docentes ao Maranhão. Os docentes eram principalmente da região Sudeste, a saber, São Paulo, Rio de Janeiro, Minas Gerais. Uma docente era proveniente do Paraná. Outros três docentes residiam no Maranhão. A diversidade de professores, quanto à instituição com a qual possuíam vínculo, as diferentes formações de cada um e, ao mesmo tempo, o interesse comum por saúde da população negra podem ser considerados também como um diferen- cial do curso. Os professores e/ou profissionais de saúde especialistas no tema saúde da população negra que integram a equipe docente são: István Varga (UFMA); Luís Eduardo Batista (IS/SES/SP); Maria de Fátima Oliveira Ferreira (HC-UFMG); Luiz Alves Ferreira (UFMA/Departamento de Patologia); Rosana Batista Monteiro (UFRRJ; UFSCar/ SP); Marco Antônio Chagas Guimarães (Instituto de Psicossomática Psicanalítica Ori-Aperê); Maria Inês da Silva Barbosa (Fundo de Desenvolvimento das Nações Unidas para a Mulher); Alaerte Leandro Martins (Secretaria de Estado da Saúde do Paraná); Joaquim Antônio César Mota (HC-UFMG); Rosilda Silva Dias (UFMA/Departamento de Enfermagem), Naila Janilde Seabra Santos (Centro de Referência e Treinamento em DST/aids).

A diversidade de formação e locais de atuação dos docentes trouxe para o curso enorme riqueza de conteúdos e experiências. Desde a elaboração da proposta a sua efetiva realização, a proposta foi construída com a participação da maioria dos docentes envolvidos, que contribuíram para a escolha dos temas a serem estudados, tendo em vista os conhecimentos específicos que cada um possuía sobre o tema, com as diferentes experiências profissionais desses docentes em relação ao tema. As formas de compreendê-lo decorriam da relação direta com o atendimento às mulheres negras à gestão de políticas e programas governamentais em Saúde da População Negra.

Desse modo, foram eleitos como temas fundamentais a serem desenvolvidos, na forma de módulos, temas que permitiriam aos cursistas compreender desde a definição das políticas de saúde pública no Brasil à definição da Política de Saúde da População Negra, suas razões e emergência, bem como as especificidades da saúde da mulher negra no contexto nacional, regional e local. A seguir apresentamos os módulos e seu desdobramento em unidades e/ou conteúdos:

3 Projeto Político Pedagógico do Curso de Especialização em Saúde da Mulher Negra. UFMA/Programa de Pós-graduação em Saúde e MEIO ambiente. Maranhão. 2007. (grifos dos autores) 


\section{Quadro I - Módulos e conteúdos da Especialização em Saúde da Mulher Negra}

\begin{tabular}{|c|c|}
\hline Módulos & Unidades/conteúdos \\
\hline I. Políticas de Saúde no Brasil & $\begin{array}{l}\text { A construção social do SUS; Plano Nacional de Saúde; } \\
\text { Controle social das políticas de saúde; Controle ético. }\end{array}$ \\
\hline II. O campo Saúde da Mulher & $\begin{array}{l}\text { Visão panorâmica sobre Saúde da Mulher; } \\
\text { Programa de Atenção Integral à Saúde da Mulher; } \\
\text { Documentos do MS sobre Atenção à Saúde da Mulher; } \\
\text { Mov. Internacional Mulher e Saúde. }\end{array}$ \\
\hline III. O campo Saúde da População Negra & $\begin{array}{l}\text { Racismo científico; quesito cor; } \\
\text { Evidências do racismo na assistência e na pesquisa em saúde; } \\
\text { Indicadores de desigualdade racial e de gênero; Epidemiologia e } \\
\text { relações raciais; } \\
\text { Campo Saúde da População Negra; } \\
\text { Política de Atenção Integral à Saúde da População Negra; } \\
\text { Racismo Ambiental. }\end{array}$ \\
\hline $\begin{array}{l}\text { IV. O campo Saúde da Mulher negra: nosologias e } \\
\text { protocolos clínicos específicos }\end{array}$ & $\begin{array}{l}\text { Morbidade e mortalidade precoce; } \\
\text { Saúde reprodutiva; } \\
\text { Esterilização e raça; } \\
\text { Violência, violência de gênero, étnico racial e saúde; } \\
\text { Hipertensão arterial; } \\
\text { Diabetes; } \\
\text { Deficiência Glicose-6-fosfato desidrogenase; } \\
\text { Mortalidade materna; } \\
\text { Mioma uterino; } \\
\text { Comitês de Prevenção à Mortalidade Materna; } \\
\text { Anemia falciforme; } \\
\text { Saúde mental; } \\
\text { A mulher negra grávida: orientações para a atenção humanizada. }\end{array}$ \\
\hline $\begin{array}{l}\text { V. Ações de prevenção em saúde da população } \\
\text { negra. }\end{array}$ & $\begin{array}{l}\text { A arte de cuidar e de curar de matriz africana; } \\
\text { Aids: feminização e enegrecimento; } \\
\text { Grupos de pessoas portadoras de diabetes: de hipertensão e de mioma } \\
\text { uterino; } \\
\text { Prevenção em Saúde Mental. }\end{array}$ \\
\hline VI. Trabalho de campo em Saúde da Mulher Negra & $\begin{array}{l}\text { Seminários de Metodologia de Pesquisa; } \\
\text { Trabalho de campo em Saúde da Mulher Negra. }\end{array}$ \\
\hline
\end{tabular}

A análise dos temas desenvolvidos em cada módulo nos possibilita afirmar a estreita relação entre o curso e o que dispõem as DCNERER e a PNSINP. Os estudos dos temas indicados no quadro, tal como afirma a Resolução CNE/CP o1/2004 em seu artigo $5^{\circ}$, colaboram para sejam corrigidas posturas e atitudes que implicam desrespeito e discriminação que, no caso da saúde, podem resultar em adoecimento e morte da população negra.

A duração dos módulos e unidades dependeu da complexidade de cada tema variando entre 12 e 16 horas para a abordagem de cada um dos temas. Desse modo, alguns módulos tiveram uma maior duração, por envolverem maior número de unidades/conteú- 
dos a serem desenvolvidos. A responsabilidade do desenvolvimento de cada módulo foi atribuída aos docentes que deveriam encaminhar com antecedência os textos e outros materiais a serem estudados pelos cursistas e, ainda, promover formas de avaliação articuladas ao desenvolvimento de trabalhos de campo e das monografias.

Como afirmamos anteriormente, os docentes do curso possuíam diferentes formações acadêmicas (das áreas de Saúde, Educação, Ciências Sociais), experiências profissionais (professores universitários, gestores, atuação nos serviços de atenção à saúde, como hospitais e clínicas, e ainda em organizações não governamentais, dentre outras) e inserção (ou não) em movimentos sociais relacionados à saúde, mulheres e população negra. Muito embora não se possa afirmar que a diversidade de formação e experiências trazida pelo corpo docente fosse suficiente para propiciar práticas interdisciplinares no desenvolvimento do curso, esta foi elemento primordial. A interdisciplinaridade esteve presente desde a proposição do curso a seu desenvolvimento, o que exigiu que alguns módulos fossem desenvolvidos por dois ou mais professores atuando em conjunto.

É possível perceber o resultado do desenvolvimento dos módulos, interdisciplinarmente, nos trabalhos de monografia, por exemplo. A preocupação em articular saúde e educação, na perspectiva da educação permanente em saúde ou da educação escolar é um elemento comum em alguns trabalhos.

Cabe observar que, apesar de o módulo VI se referir ao trabalho de campo (Seminários de metodologia de pesquisa e trabalho de campo), este foi desenvolvido, no decorrer do curso, de forma a se articular com o desenvolvimento dos módulos teórico-práticos e propiciar melhores condições para a construção dos projetos de pesquisa e seu desenvolvimento. Ainda assim, como veremos mais adiante, muitas foram as dificuldades para a finalização das monografias pelos cursistas.

\section{Temas dos projetos de pesquisa: encaminhamentos e dificuldades}

Desde o início do curso os cursistas foram estimulados a pensarem em seus projetos de pesquisa.
A diferença entre os dois grupos, os de Pinheiro e os de São Luís, com maior número de professores no primeiro e de profissionais de saúde no segundo, resultou em maior número de projetos ligados mais diretamente à saúde pelo grupo de São Luís. Os temas foram discutidos coletivamente nos encontros do módulo VI, em que eram combinadas aulas expositivo-dialogadas de metodologia de pesquisa e oficina de elaboração de projetos de pesquisa. A docente responsável pelo módulo foi a principal articuladora, à distância e presencialmente, das ações de desenvolvimento dos pré-projetos, e a indicação dos possíveis orientadores de acordo com os temas propostos.

Os cursistas que optaram por serem orientados por professores de fora do Maranhão realizaram seus trabalhos sob orientação à distância na maior parte das vezes. Alguns foram acompanhados ainda pelos professores da UFMA que, para auxiliarem nessa tarefa, apoiaram os cursistas sempre em articulação com seus orientadores.

Observamos ao longo do desenvolvimento do curso que a fase da elaboração das monografias foi a mais difícil. Infelizmente, mesmo com boas propostas de pesquisa, muitos desistiram de realizá-las, possivelmente pela dificuldade com a realização da pesquisa e escrita do texto sem a presença concreta de seus orientadores. Os cursistas mais jovens, com alguma experiência no uso de ferramentas da informática (Skype, e-mails, envio de arquivos, e outros), ou que por alguma razão conseguiram estar em maior contato com seus orientadores foram os que finalizaram suas monografias.

O quadro 2 se refere aos temas eleitos pelos cursistas para a elaboração de seus projetos de pesquisa.

Um número significativo de cursistas realizou a elaboração dos projetos de pesquisa e, para tanto, além da participação nas aulas, realização de leituras, desenvolveram pesquisa inicial, documental ou empírica. Observa-se que a maioria dos projetos apresenta temas diretamente relacionados aos problemas de saúde das mulheres negras das localidades expandidas onde o curso se desenvolveu, com questões vividas no cotidiano de muitas das cursistas, a exemplo do alto índice de mulheres negras histerectomizadas, hiperten- 


\section{Quadro 2 - Temas/títulos de projetos de pesquisa apresentados}

Projetos de pesquisa

I. A cor da mortalidade materna em São Luís - Maranhão

2. A realidade das mulheres falciforme com úlcera de membro atendidas no centro de referência para o diagnóstico e tratamento de anemia falciforme em São Luís/MA

3. Análise da promoção à saúde da mulher negra no Povoado Castelo município de Alcântara/MA

4. A abordagem de Saúde da População Negra nos cursos de graduação em medicina das Universidades do Estado do Maranhão

5. Mulheres negras: uma análise da relação entre escolaridade, hábitos de vida e conhecimento sobre hipertensão arterial

6. Mortalidade de mulheres negras no Brasil: um estudo de revisão bibliográfica

7. Avaliação da assistência pré-natal em mulheres negras atendidas em uma unidade básica de saúde

8. 0 resgate histórico das mulheres que contribuíram para o nascimento de crianças em regiões interioranas, ou seja, mulheres parteiras

9. Análise das causas de mortalidade materna em negras no Hospital Materno-Infantil de Pinheiro, entre 2005-2009

10. Hipertensão arterial sistêmica segundo o quesito cor: o que nos diz o sistema de cadastramento de informações em saúde do município de São Luís/MA

II. Estudo descritivo sobre a aids em mulheres negras no município de Pinheiro/MA

12. Parto domiciliar: análise situacional do parto domiciliar em mulheres negras que vivenciaram essa experiência no município de São Vicente Ferrer/MA

13. Prevalência de gravidez na adolescência em mulheres negras e não negras no hospital materno infantil, nos anos entre 2005 e 2009, no município de Pinheiro/MA

14. A realidade de mulheres histerectomizadas no município de Viana/MA

15. 0 processo de construção da política estadual de saúde da população negra no Maranhão - um resgate histórico da agenda política do movimento negro, de mulheres negras e de terreiros de religiões de matriz africana

16. A importância sobre a identificação da identidade étnico-racial no serviço de pronto atendimento do Hospital Frei Sinibaldi

17. Histórias de vida de mulheres negras hipertensas da comunidade Curitiba, em Palmeirândia/MA

18. Revisão bibliográfica sobre hipertensão arterial sistêmica entre negros

19. Mulheres negras jovens na rede pública de ensino e aspectos da vulnerabilidade em São Luís do Maranhão

20. Uma análise da mortalidade materna de mulheres negras no município de Palmeirândia/MA

21. Perfil epidemiológico de mulheres negras atendidas no Hospital Universitário Materno Infantil de São Luís/MA

22. A informação da juventude negra sobre o HIV em uma escola no município de Pinheiro/MA

23. 0 conhecimento (informação) das mulheres negras sobre a importância do exame preventivo do colo do útero em um centro de saúde da São Luís

24. Os pacientes falcêmicos na prática da Educação Física em São Luís

25. 0 impacto do racismo na saúde mental das mulheres negras: um estudo de revisão bibliográfica

26. Pensando na importância do atendimento para adolescentes negras e não negras na área 59 da ESF (São Luís)

27. Avanços e desafios da política de atenção integral à saúde da população negra em São Luís

28. Humanização na assistência pré-natal às mulheres negras no município de Itapecuru-Mirim

29. Perfil da mortalidade da mulher negra no estado do Maranhão em 2008

30. Implementação da política de atenção integral à saúde da população negra em São Luís

31. A população negra e cultos de matriz afro na obra de Lacan

32. 0 lazer e a qualidade de vida de mulheres grávidas e não grávidas com anemia falciforme atendidas no Hemomar

33. Análise das causas de mortalidade materna em mulheres negras ocorridas no Hospital Materno Infantil de Pinheiro no período 2005-2009

34. Nascido em casa: o papel das parteiras na comunidade de Jamary dos Pretos

35. Histerectomia em mulheres negras: uma necessidade ou preconceito

36. As influências socioeconômicas e aspectos socioambientais nos quilombos de Mondego e Frechal em Mirinzal

37. Nível de informação dos agentes comunitários de saúde em Pedro do Rosário 
sas. Observa-se, ainda, a forte preocupação com questões relacionadas à saúde da mulher negra e às estratégias tradicionais de promoção de saúde, a exemplo de projetos que apontam para o estudo do parto domiciliar, saúde e terreiros, saúde mental e religiões de matriz africana. E ainda o interesse em se pesquisar a saúde das mulheres negras de determinadas comunidades, a exemplo de quilombos, muito presentes no Maranhão.

Ainda que poucos trabalhos tenham chegado à defesa (apenas oito deles), nossa avaliação é de que o curso em muito contribuiu para a efetiva implementação das DCNERER e da PNISPN.

Entre os trabalhos finalizados gostaríamos de destacar dois deles, referentes aos projetos anteriormente listados: Mulheres negras jovens na rede pública de ensino e aspectos da vulnerabilidade em São Luís, de Maria Lucia Gato de Jesus e, Avaliação da assistência pré-natal em mulheres negras atendidas em uma unidade de saúde da família, de Ilka Kassandra Pereira Belfort ${ }^{4}$.

0 primeiro trabalho trouxe uma dupla contribuição ao debate sobre saúde da mulher negra. Professora de Ciências e Biologia da rede pública, na cidade de São Luís, e militante do movimento negro, Maria Lucia G. de Jesus tinha como preocupação a situação vivenciada cotidianamente nas escolas em relação às jovens negras. Sua observação era de que as jovens, principalmente negras, iniciavam sua vida sexual sem informações, engravidavam cedo e, por fim, tinham dificuldade para se manter na escola. Sua pesquisa mostra a escola como espaço privilegiado para obtenção de informações sobre saúde, porém, não formalmente. Ou seja, não são os professores que informam as jovens. A escola é espaço de circulação de informações entre colegas da escola, que na maioria das vezes não possuem senão suas próprias experiências para dividir. Não há ainda diálogo entre escola e unidades básicas de saúde, encaminhamento das jovens para atendimento.

O trabalho possibilitou o retorno dos resultados à escola, indicando a necessidade de se rever o projeto político pedagógico, incluindo as estratégias que contribuam para melhor formação das jovens e, consequentemente, evitar sua evasão. E ainda, vivenciar sua sexualidade de forma mais positiva, valorizando suas identidades de gênero e racial.

O trabalho de Ilka Belfort, da mesma forma, trouxe contribuições sua comunidade, especialmente no que tange a melhor compreensão da realidade sobre nascimento e parto em Icatú, município do estado do Maranhão. Ao investigar como as mulheres negras eram assistidas durante o pré-natal e parto, a cursista evidencia as negligências perpassadas pelo racismo, que levam as mulheres negras à morte. Fato esse que, infelizmente, é uma realidade nacional. Mas reconhecer como os processos discriminatórios operam torna possível atuar para modificá-los. Reconhecer as especificidades que se põem para as mulheres negras grávidas contribui para evitar as diferenças de mortalidade entre negras e não negras, bem como previamente saber como agir.

\section{Considerações finais}

O texto da Política Nacional de Saúde Integral da População Negra determina "a inclusão dos temas racismo e saúde da população negra nos processos de formação e educação permanente dos trabalhadores da saúde e no exercício do controle social na saúde" (Brasil, 2009).

As DCNERER afirmam que a inclusão de conteúdos relacionados à educação para as relações étnico-raciais e o ensino de história e cultura afro-brasileira e africana tem por meta "promover a educação de cidadãos atuantes e conscientes no seio da sociedade multicultural e pluriétnica do Brasil, buscando relações sociais positivas, rumo à construção de nação democrática" (Brasil, 2004a).

O desenvolvimento do Curso de Especialização em Saúde da Mulher Negra, como pudemos mostrar neste ensaio, pode ser tomado como uma referência na atenção ao disposto nas políticas de ação afirmativa na educação e na saúde pública representadas pela Portaria $n^{0}$ 992/2009 e pela Resolução CNE 01/2004 que institui as DCNERER. Articula diferentes profissionais, de diferentes áreas e formação, especialmente da saúde e da educação, docentes e discentes/cursistas, identifica temas e literatura relacionados direta e indiretamente com saúde da

4 Os títulos se referem aos projetos, e não ao título final das monografias. 
população negra/mulheres negras, indica caminhos de valorização dos saberes trazidos pelos movimentos sociais e saberes tradicionais especialmente vindos das religiões de matriz africana. 0 resultado dos projetos e monografias, especialmente por sua relação com o cotidiano da escola, das unidades de atendimento em saúde e comunidades, demonstram ainda, mais que o alcance dos objetivos propostos pelo curso, que são evidências de que é possível, por meio da educação permanente, construir estratégias para reverter positivamente práticas instaladas, amalgamadas por séculos nos equipamentos públicos, na saúde e na educação, viabilizando a construção de outra forma de estabelecer relações sociais, nesse caso, no estado do Maranhão. Relações essas que, modificadas, resultam em qualidade de vida, especialmente para a população negra.

\section{Referências}

BARREIRA, M. C. R. N.; CARVALHO, M. C. B. (Org.). Tendências e perspectivas na avaliação de políticas e programas sociais. São Paulo: IEE/ PUC-SP, 2001.

BATISTA, L. E. Mulheres e homens negros: saúde, doença e morte. 2002. Tese (Doutorado em Sociologia) - Faculdade de Ciências e Letras da Universidade Estadual Paulista "Julio de Mesquita Filho”, Araraquara, 2002.

BATISTA, L. E.; KALCKMANN, S. (Org.). Seminário saúde da população negra: estado de São Paulo 2004. São Paulo: Instituto de Saúde, 2005.

BATISTA, L. E.; WERNECK J.; LOPES, F. (Org.). Saúde da população negra. 2. ed. Brasília, DF: Associação Brasileira de Pesquisadores Negros, 2012.

BRASIL. Ministério da Educação. Conselho Nacional de Educação. Parecer CNE/CP oo3/2004, de 10 de março de 2004. Diretrizes Curriculares Nacionais para a Educação das Relações Étnicoraciais e para o Ensino de História e Cultura Afro-Brasileira e Africana. Diário Oficial da União, Brasília, DF, 19 maio 2004a. Seção 1, p. 19. BRASIL. Ministério da Educação. Conselho Nacional de Educação. Resolução nº 1 , de 17 de junho de 2004. Diretrizes Curriculares Nacionais para a Educação das Relações Étnico-raciais e para o Ensino de História e Cultura AfroBrasileira e Africana. Diário Oficial da União, Brasília, DF, 22 jun. 2004b. Seção 1, p. 11.

BRASIL. Ministério da Educação. Secretaria da Educação Continuada, Alfabetização e Diversidade. Orientações e ações para educação das relações étnico/raciais. Brasília, DF: SECAD, 2006.

BRASIL. Ministério da Saúde. Portaria nº 992, de 13 de maio de 2009. Institui a Política Nacional de Saúde Integral da População Negra. Diário Oficial da União, Brasília, DF, 14 maio 2009. Seção 1, p. 31.KALCKMANN, S. Racismo institucional: um desafio para a equidade no SUS? Saúde e Sociedade, São Paulo, v. 16, n. 2, p. 146-155, 2007.

CUNHA, M. E. de P. Mortalidade infantil e raça: as diferenças da desigualdade. [s. d.]. Disponível em: <http://www.saude.sp.gov.br/resources/ses/ perfil/profissional-da-saude/grupo-tecnico-deacoes-estrategicas-gtae/saude-da-populacaonegra/artigos-e-teses/mortalidade_infantil_e_ raca_as_diferencas_da_desigualdade.pdf $>$. Acesso em: 8 ago. 2016.

LOPES, F. Mulheres negras e não negras vivendo com HIV/Aids no estado de São Paulo: um estudo sobre suas vulnerabilidades. 2003. Tese (Doutorado em Epidemiologia) - Faculdade de Saúde Pública da Universidade de São Paulo, São Paulo, 2003.

LOPES, F. Conceitos e aplicabilidades dos determinantes sociais da saúde-DSS nas políticas do SUS. In: FÓRUM ENFRENTANDO O RACISMO INSTITUCIONAL PARA PROMOVER SAÚDE INTEGRAL DA POPULAÇÃO NEGRA NO SUS, 2012, Brasília, DF. Relatório final... Brasília, DF, 2012, p. 21-24. Disponível em: <http://www.unfpa.org. br/Arquivos/relatorio_forum_enfrentamento_ racismo.pdf >. Acesso em: 7 jul. 2016.

LOPES, F.; BUCHALLA, C. M.; AYRES, J. R. C. M. Mulheres negras e não negras e vulnerabilidade ao HIV/Aids no estado de São Paulo, Brasil.

Revista de Saúde Pública, São Paulo, v. 41, p. 3946, 2007. Suplemento 2. Disponível em: <http:// 
www.scielo.br/pdf/rsp/v41s2/5952.pdf >. Acesso em: 7 jul. 2016.

MONTEIRO, R. B. A educação para as relações étnicoraciais em um curso de Pedagogia: estudo de caso sobre a implantação da Resolução CNE/CP o1/2004. 2010. Tese (Doutorado em Educação) - Universidade Federal de São Carlos, São Carlos, 2010.

MONTEIRO, R. B. Orientações e experiências para a implementação da Política Nacional de Saúde Integral da População Negra no âmbito da formação e Educação Permanente em Saúde. 2011. Documento elaborado para o Comitê Técnico de Saúde da População Negra/Secretaria de Gestão Estratégica e Participação do Ministério da Saúde.

MUNANGA, K. Teoria social e relações raciais no Brasil contemporâneo. Cadernos Penesb, Niterói, n. 12, p. 169-203, 2010.
OLIVEIRA, F. Saúde da população negra: Brasil ano 2001. Brasília, DF: Organização Pan-Americana da Saúde, 2003.

OLIVEIRA, I. (Org.). Relações raciais no contexto social, na educação e na saúde: Brasil, Cuba, Colômbia e África do Sul. Rio de Janeiro: Quartet, 2012.

SISTEMA NAÇÕES UNIDAS. Subsídios para o debate sobre a Política Nacional de Saúde da População Negra: uma questão de equidade. Brasília, DF, 2001.

WERNECK, J. Iniquidades raciais em saúde e políticas de enfrentamento: as experiências do Canadá, Estados Unidos, África do Sul e Reino Unido. In: BRASIL. Fundação Nacional de Saúde. Saúde da população negra no Brasil: contribuições para a promoção da equidade. Brasília, DF: Funasa, 2005, p. 315-386. 